\title{
AN ELECTRON-MICROSCOPIC STUDY OF THE DEFORMATION STRUCTURE OF THE 12Kh18N10T STEEL AFTER EXPLOSIVE LOADING IN SPHERICAL SYSTEMS
}

\author{
A. V. Dobromyslov*, N. I. Taluts \\ M.N. Miheev Institute of Metal Physics of Ural Branch of Russian Academy of Sciences, 18 S. Kovalevskoy st., \\ Ekaterinburg, Russian Federation \\ *Corresponding author. E-mail: Dobromyslov@imp.uran.ru; address for correspondence: 18, ul. S. Kovalevskoy,
620990, Ekaterinburg, Russian Federation. Tel.: +7 343 3783824; Fax: +7 343 3745244
}

Optical metallography, transmission electron microscopy and microhardness measurements are used to investigate the deformed structure of retained shells made of the $12 \mathrm{Kh} 18 \mathrm{~N} 10 \mathrm{~T}$ steel after explosive loading. It has been established that the high-rate plastic deformation of the steel under this loading occurs both by slipping and twinning. It is shown that there is a strong localization of deformation resulted in the formation of rough traces of slip. The high pressure at the shock wave front results in the fact that the critical shear stress in one grain is achieved in several slip systems simultaneously, irrespective of the Schmid factor. Therefore, several nonequivalent systems become active slip systems at once. Microtwins form large clusters in which they mainly belong to one or two systems of twinning. The average thickness of microtwins is $\sim 30-40 \mathrm{~nm}$. Polymorphic $\gamma \rightarrow \alpha$ transformation has been revealed under explosive loading. The $\alpha$-phase is observed in the form of fine precipitates. It has been found that the microhardness almost doubles after shock loading, as compared with that of the initial state.

Keywords: 12Kh18N10T steel, shock waves, high-rate plastic deformation, structure.

DOI: $10.17804 / 2410-9908.2015 .5 .109-117$

\section{References}

1. Meyers M.F., Murr L.E. Defect generation in shock-wave deformation. In: M. A. Meyers, L. E. Murr, eds. Shock waves and high-strain-rate phenomena in metals. New York, Plenum Press, 1981, pp. 487-530.

2. Sencer B.H., Maloy S.A., Gray III G.T. The influence of explosive-driven shock prestraining at $35 \mathrm{GPa}$ and of high deformation on the structure/property behavior of $316 \mathrm{~L}$ austenitic stainless steel. Metallurgical and Materials Transactions A: Physical Metallurgy and Materials Science, 2005, vol. 36, iss. 7, pp. 1825-1831. DOI: 10.1016/j.actamat.2005.03.037.

3. Lee Woel-Shyan, Lin Chi-Feng. Comparative study of the impact response and microstructure of 304L stainless steel with and without prestrain. Metallurgical and Materials Transactions A, 2002, vol. 33, issue 9, pp. 2801-2810. DOI: 10.1007/s11661-002-0265-4.

4. Murr L.E., Staudhammer K.P., Hecker S.S. Effects of Strain State and Strain Rate on Deformation-Induced Transformation in 304 Stainless Steel: Part II. Microstructural Study. Metallurgical Transactions A, 1982, vol. 13, iss. 4, pp. 627-635. DOI: 10.1007/BF02644428.

5. Malloy S.A., Gray III G. T., Cady C.M., Rutherford R.W., Hihson R.S. The influence of explosive-driven "taylor-wave" shock prestraining on the structure/property behavior of 304 stainless steel. Metallurgical and Materials Transactions A, 2004, vol. 35, iss. 9, pp. 2617-2624. DOI: $10.1007 / \mathrm{s} 11661-004-0207-4$.

6. $\quad$ Firraro D., Matteis P., Scavino G., Ubertalli G., Ienco M. G., Pellati G., Piccardo P., Pinasco M.R., Stagno E., Montanari R., Tata M.E., Brandimarte G., Petralia S., Mechanical twins in 304 stainless steel after small-charge explosion. Materials Science and Engineering: A, 2006, vol. 424, iss. 1-2, pp. 23-32. DOI: 10.1016/j.msea.2006.02.036.

7. Kozlov E.A., Brichikov S.A., Boyarnikov D.S., Kuchko D.P., Degtyarev A.A. Special features in convergence dynamics of steel shells under their explosive loading. Results of laser- 
open-access journal

interferometric measurements. The Physics of Metals and Metallography, 2011, vol. 112, iss. 4, pp. 389-404. DOI: 10.1134/S0031918X11040259.

8. Rutkowska-Gorczyca M., Podrez-Radziszwska M., Kajtoch J. Corrosion resistance and microstructure of steel AISI 316L after cold plastic deformation. Metallurgy and foundry engineering, 2009, vol. 35 , no. 1 , pp. 35-42.

9. Borodin E.N., Atroshenko S. A., Mayer A.E. Distribution of dislocations and twins in copper and 18Cr-10Ni-Ti steel under shock-wave loading. Technical Physics, 2014, vol. 59, iss. 8, pp. 1163-1170. DOI: 10.1134/S1063784214080076.

10. Bogers A.J., Burgers W.G. Partial dislocations on the $\{110\}$ planes in the B.C.C. lattice and the transition of the F.C.C. into the bec lattice. Acta Metallurgica, 1964, vol. 12, iss. 2, pp. 255-261. DOI: 10.1016/0001-6160(64)90194-4.

11. Talonen J., Hanninen H. Formation of shear bands and strain-induced martensite during plastic deformation of metastable austenitic stainless steels. Acta Materialia, 2007, vol. 55, iss. 18, pp. 6108-6118. DOI: 10.1016/j.actamat.2007.07.015.

12. Zel'dovich V.I., Kheifets A.E., Frolova N.Yu., Muzyrya A.K., Simonov A.Yu. Formation of martensite in austenitic steel upon loading by quasi-spherical converging shock waves. The Physics

of Metals and Metallography, 2013, vol. 114, iss. 12, pp. 1031-1037. DOI: $10.1134 / \mathrm{S} 0031918 X 13120090$. 
Подана в журнал: 17.09 .2015

УДК 539.89

DOI: $10.17804 / 2410-9908.2015 .5 .109-117$

\title{
ЭЛЕКТРОННО-МИКРОСКОПИЧЕСКОЕ ИССЛЕДОВАНИЕ ДЕФОРМАЦИОННОЙ СТРУКТУРЫ СТАЛИ 12Х18Н10Т ПОСЛЕ ВЗРЫВНОГО НАГРУЖЕНИЯ В СФЕРИЧЕСКИХ СИСТЕМАХ
}

\author{
А. В. Добромыслов*, Н. И. Талуц \\ Федеральное государственное бюджетное учреждение науки Институт физики металлов имени \\ М.Н. Михеева Уральского отделения Российской академии наук, ул. С. Ковалевской, 18, Екатеринбург, \\ Российская Федерациия \\ *Ответственный автор. Электронная почта: Dobromyslov@,imp.uran.ru; адрес для переписки: 620049, \\ ул. С. Ковалевской, 18, Екатеринбург, Российская Федерация. Телефон +7 (343) 378-38-24; \\ факс: +7 (343) 374-52-44
}

Изучена деформационная структура сохраненных оболочек из нержавеющей аустенитной стали 12Х18Н10Т после взрывного нагружения. Установлено, что высокоскоростная пластическая деформация стали в условиях ударного нагружения осуществляется как скольжением, так и двойникованием. Показано, что в процессе деформации происходит сильная локализация деформации, приводящая к образованию грубых следов скольжения. Высокое давление на фронте ударной волны приводит к тому, что критическое напряжение сдвига в одном зерне достигается сразу же в нескольких системах, независимо от фактора Шмида, в результате чего активными системами скольжения становятся несколько неэквивалентных систем. Обнаружено, что микродвойники формируют большие скопления, в которых они в основном принадлежат к одной или двум системам двойникования. Средняя толщина микродвойников составляет 30-40 нм. Обнаружено, что при взрывном нагружении протекает полиморфное $\gamma \rightarrow \alpha$ превращение. Образующаяся $\alpha$-фаза наблюдается в виде мелких выделений. Установлено, что значение микротвердости после ударного нагружения увеличилось примерно в 2 раза по сравнению с исходным состоянием.

Ключевые слова: сталь 12Х18Н10T, ударное нагружение, высокоскоростная пластическая деформачия, структура.

\section{1. Введение}

Нержавеющая сталь 12X18Н10Т находит широкое применение в науке и технике, где требуются высокие антикоррозионные свойства и повышенная стойкость к действию различных температур. Ударное воздействие принадлежит к числу важных упрочняющих обработок материалов и в настоящее время широко используется в практике. Образующаяся в процессе ударного нагружения деформационная структура зависит от величины давления на фронте ударной волны, продолжительности импульса ударного воздействия и скорости деформации.

Изучение влияния ударных волн на механические свойства и структуру нержавеющих аустенитных сталей проводилось в ряде работ [1-6]. В структуре этих сталей после ударного нагружения было обнаружено присутствие полос сдвига, деформационных двойников, а при низких давлениях - также дефектов упаковки [1]. Отмечалось, что появление того и другого типа деформационных дефектов сильно зависит от условий нагружения [3]. Следует отметить, что при проведении таких исследований нагружение образцов проводилось плоскими ударными волнами. В работе [7] были изучены особенности динамики схождения высокорасположенных по радиусу оболочек из аустенитной нержавеющей стали 12 X18Н10Т в сферических взрывных системах. 
Цель данной работы - исследование деформационной структуры и механизма деформации нержавеющей стали 12X18Н10T (режим нагружения 4 [7]), а также особенностей протекания в них фазового $\gamma \rightarrow \alpha$ превращения.

\section{2. Материал и методика}

Исследование проводилось на оболочках, изготовленных из нержавеющей стали 12Х18Н10Т (табл. 1).

Таблица 1 - Химический состав исследуемой стали, мас. \%

\begin{tabular}{|c|c|c|c|c|c|c|c|c|c|}
\hline Fe & $\mathrm{C}$ & $\mathrm{Cr}$ & $\mathrm{Ni}$ & $\mathrm{Ti}$ & $\mathrm{Mn}$ & $\mathrm{Cu}$ & $\mathrm{Si}$ & $\mathrm{P}$ & $\mathrm{S}$ \\
\hline Oсн. & $\leq 0,12$ & $17,0-19,0$ & $9,0-11,0$ & $0,6-0,8$ & $\leq 2,0$ & $\leq 0,3$ & $\leq 0,8$ & $\leq 0,035$ & $\leq 0,02$ \\
\hline
\end{tabular}

Нагружение оболочек проводилось по 4-му режиму, описанному в работе [7]. Толщина 1-й оболочки составляла 2,95 мм, а 2-й - 4,04 мм. Из каждой оболочки были вырезаны шлифы для проведения металлографического исследования и измерения микротвердости, а также фольги для электронно-микроскопического изучения структуры. Шлифы готовили механически и затем травили в реактивах следующего состава: 5г $\mathrm{FeCl}_{3}+15$ мл $\mathrm{HCl}+50$ мл $\mathrm{H}_{2} \mathrm{O} ; 2$ г пикриновой кислоты +3 мл $\mathrm{HCl}+50$ мл этилового спирта или электролитически в $10 \%$-м водном растворе хромового ангидрида при напряжении 15-20 в. Утонение фольг осуществлялось электролитически в растворе хромового ангидрида (100 г) в ортофосфорной кислоте (860 мл) при напряжении 25-30 в.

Металлографические исследования проводили с помощью оптического микроскопа Neophot-32, рентгеноструктурный анализ - на дифрактометре ДРОН-3 с использованием $\mathrm{Cu}$ $\mathrm{K}_{\alpha}$-излучения и графитового кристалл-монохроматора, исследование микроструктуры - на просвечивающем электронном микроскопе JEM 200CX, измерение микротвердости - на микротвердомере ПМТ-3 при нагрузке 0,49 Н.

\section{3. Результаты и обсуждение}

Аттестация исходного состояния стали 12X18Н10T была проведена на образцесвидетеле, вырезанном из исходной заготовки и не претерпевшем ударно-волнового нагружения. Из рентгенографических данных следует, что сталь в исходном состоянии состоит только из $\gamma$-фазы, причем линии дублета К $\alpha$, расположенные на дифрактограммах в больших углах $\theta$, расщепляются на линии $K \alpha_{1}$ и К $\alpha_{2}$. Этот факт свидетельствует о достаточно высоком совершенстве структуры сплава. На рис. 1 показана структура исследуемой стали в исходном состоянии. Во многих зернах видно присутствие двойников отжига. При металлографическом исследовании обнаруживаются выделения $\alpha$-фазы ( $\delta$-феррита) в виде протяженных цепочек. Определение содержания $\delta$-фазы по величине намагниченности показало, что ее в стали в исходном состоянии $\sim 2 \%$, поэтому присутствие $\delta$-фазы рентгенографически не обнаруживается. Микротвердость стали в исходном состоянии равна $\mathrm{H}_{\mu}=1,88 \pm 0,08$ ГПа.

Полученные результаты свидетельствуют о том, что разная исходная толщина оболочек при одинаковом режиме нагружения не оказывает существенного влияния ни на структуру, ни на микротвердость сохраненных оболочек.

Измерение микротвердости проводилось на поперечном сечении образцов. Никакой разницы в изменении микротвердости в зависимости удаления от внешней поверхности полусфер не было обнаружено. По сравнению с микротвердостью в исходном состоянии ее значение после ударного нагружения в обеих оболочках увеличилось примерно в 2 раза.

При металлографическом изучении структуры образцов после ударного нагружения обнаружено, что форма исходного $\gamma$-зерна сильно изменяется - уменьшается поперечный размер зерна и сильно увеличивается его размер в радиальном направлении. При этом внутри зерен наблюдается присутствие большого числа полос сдвига, образующихся по несколь- 
ким системам (рис. 2). Такие полосы сдвига также наблюдаются в аустенитной стали после холодной пластической деформации [8]. Имеющиеся в исходном состоянии тонкие прослойки $\delta$-феррита сохраняются (рис. 2 б).

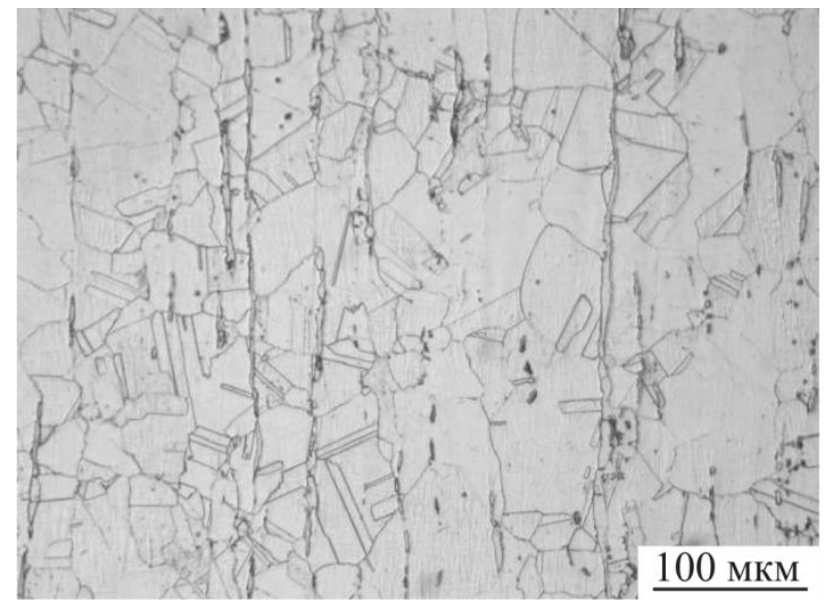

Рис. 1. Структура стали 12Х18Н10Т в исходном состоянии

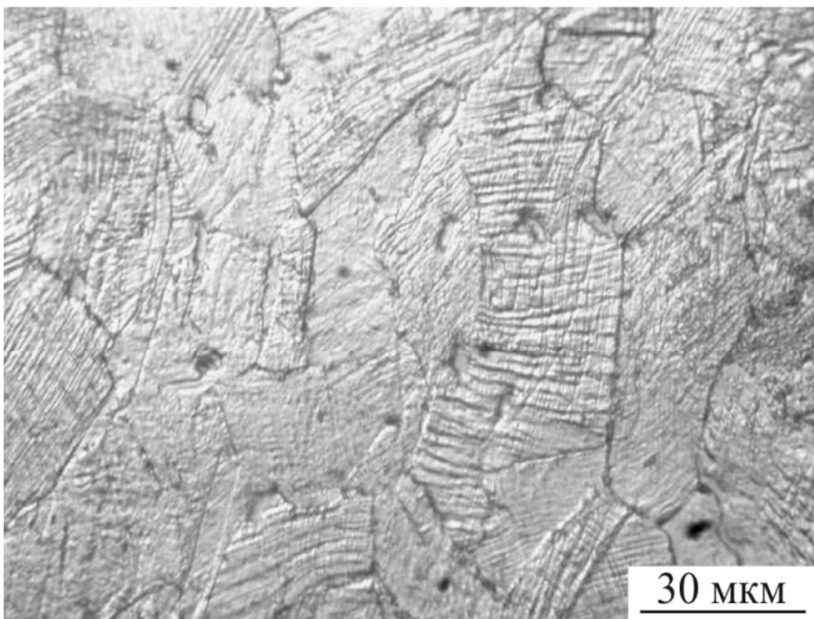

$a$

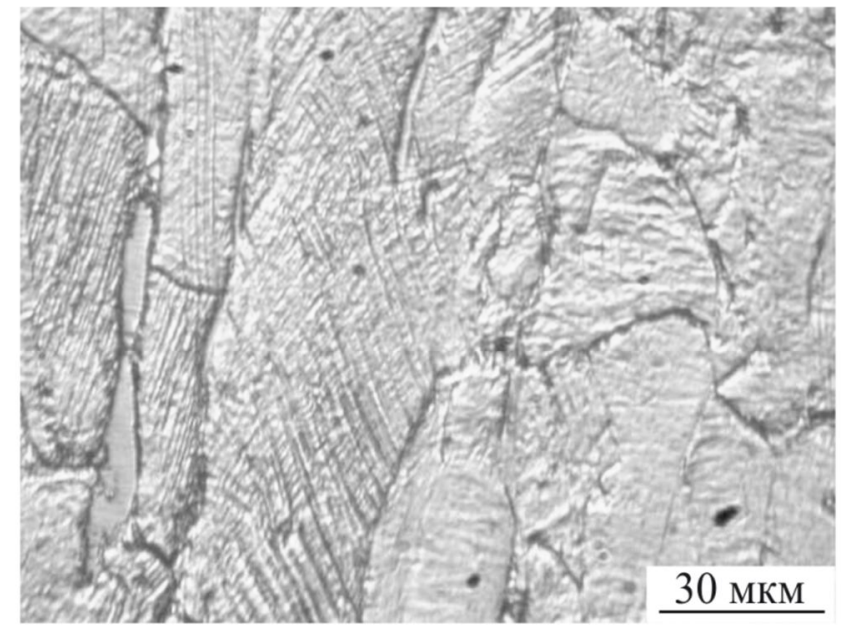

б

Рис. 2. Структура стали 12Х18Н10Т после нагружения:

$a$ - полосы скольжения по нескольким системам; $\sigma$ - сохранение зерен $\delta$-феррита

Характер деформационной структуры свидетельствует о том, что высокоскоростная пластическая деформация нержавеющей стали на макроуровне осуществляется скольжением, причем наличие в структуре грубых следов скольжения указывает на сильную локализацию пластической деформации. Одновременное действие нескольких систем скольжения в одном зерне в условиях ударного нагружения указывает на то, что приведенное сдвиговое напряжение достигает критического сдвигового напряжения в нескольких системах независимо от величины в них фактора Шмида.

При электронно-микроскопическом изучении деформационной структуры стали после нагружения наблюдается присутствие большого количества микродвойников деформации, которые образуются по плоскостям $\{111\} \gamma$-фазы. (рис. 3 и 4). В работе [9] при изучении этой стали после ударно-волнового нагружения ударником со скоростью 229 м/с также наблюдалось образование двойников. В большинстве случаев микродвойники формируют большие скопления, в которых они в основном принадлежат к одной системе двойникования (рис. 3 и $4 a$ ). Средняя толщина таких микродвойников составляет примерно 30-40 нм. О ма 
лой толщине микродвойников свидетельствует также присутствие на микроэлектронограммах тонких диффузных штрихов, связывающих двойниковые и матричные отражения (рис. 3 б). Скопления микродвойников иногда пересекают микродвойники, принадлежащие другим системам двойникования. Такие микродвойники имеют существенно большие размеры и не образуют скоплений (рис. 4 б).

Кроме скоплений микродвойников, принадлежащих к одной системе двойникования, в структуре наблюдаются участки со скоплениями микродвойников, принадлежащих к двум системам (рис. 4 в, г). Среднее расстояние между микродвойниками составляет в этом случае 0,3-0,5 мкм. Присутствие в деформационной структуре большого количества микродвойников указывает на то, что наряду со скольжением высокоскоростная пластическая деформация стали $12 \mathrm{X} 18 \mathrm{H} 10 \mathrm{~T}$ в процессе взрывного нагружения на микроуровне осуществляется двойникованием.

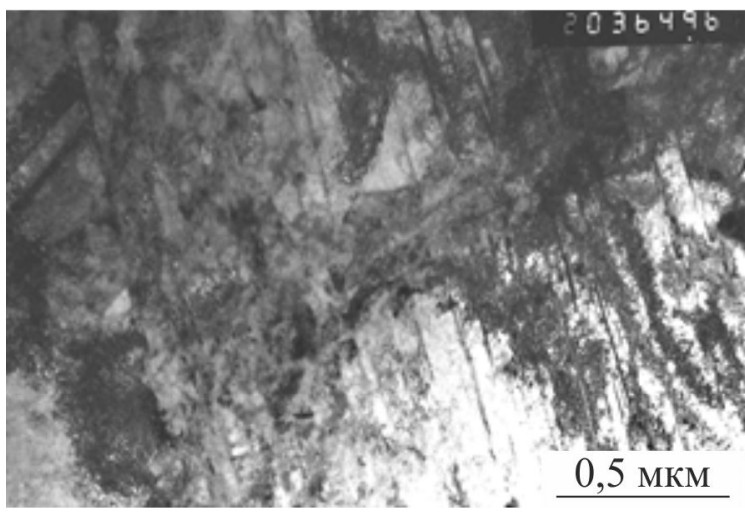

$a$

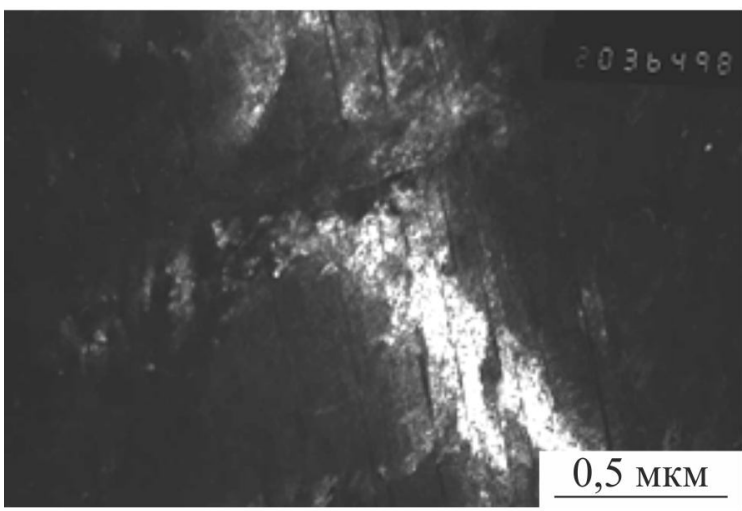

B

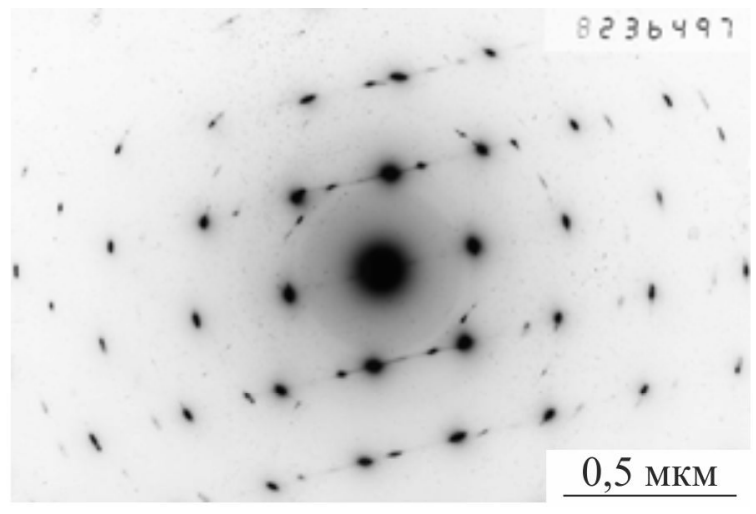

$\sigma$

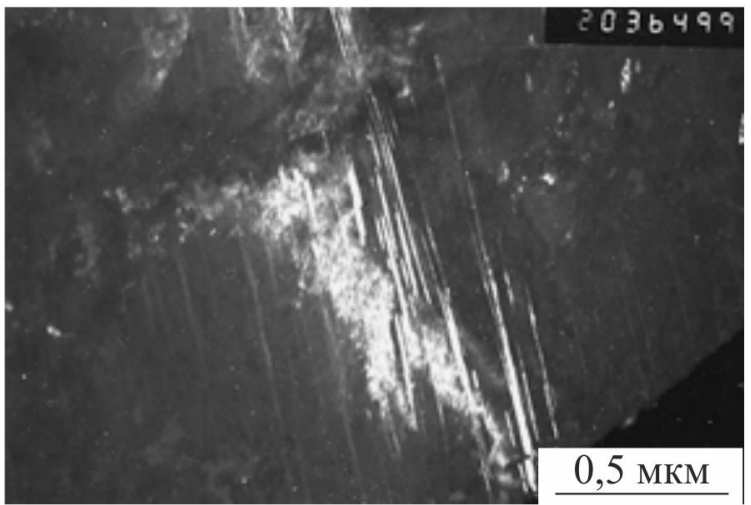

2

Рис. 3. Тонкие микродвойники в стали $12 \mathrm{X} 18 \mathrm{H} 10 \mathrm{~T}$ после нагружения: $a$ - светлопольное изображение; $\sigma$ - микроэлектронограмма к $(a)$, ось зоны основной ориентации [110];

в - темнопольное изображение, полученное в матричном рефлексе $(1 \overline{1} \overline{1})_{\gamma}$; 2 - темнопольное изображение, полученное в рефлексе двойника (002)

Характерной особенностью деформационной структуры стали после ударного нагружения является отсутствие дефектов упаковки, образование которых происходит в этой стали при небольших величинах давления [1].

Увеличение намагниченности сохраненных оболочек свидетельствует о том, что в процессе взрывного нагружения протекает $\gamma \rightarrow \alpha$ превращение. На светлопольных изображениях структуры присутствие $\alpha$-фазы обнаружить практически невозможно (рис. $5 a$ ). Однако на микроэлектронограммах в некоторых случаях присутствуют слабые рефлексы, принадлежащие $\alpha$-фазе (рис. 5 б, г). На темнопольных снимках, полученных в рефлексах $\alpha$-фазы, 
наблюдаются мелкие выделения размером 20-30 нм, несколько вытянутые в направлении распространения микродвойников (рис. 5 в). Из-за того, что на микроэлектронограммах присутствуют только отдельные рефлексы $\alpha$-фазы, определить ориентационные соотношения между $\gamma$-фазой и образующейся $\alpha$-фазой сложно. Можно только установить, что плоскость (100) $\alpha$-фазы параллельна плоскостям (110) $\gamma$-фазы. Выделения такой формы ранее наблюдались в [2]. Авторы этой работы назвали их мартенситными зародышами.

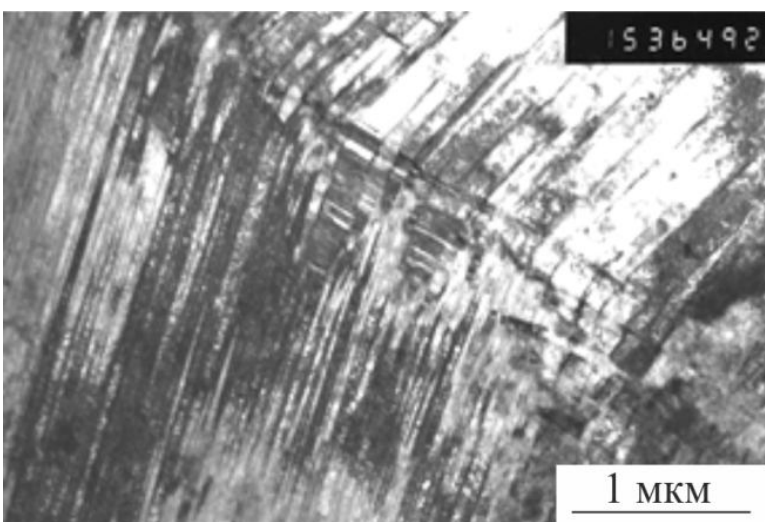

$a$

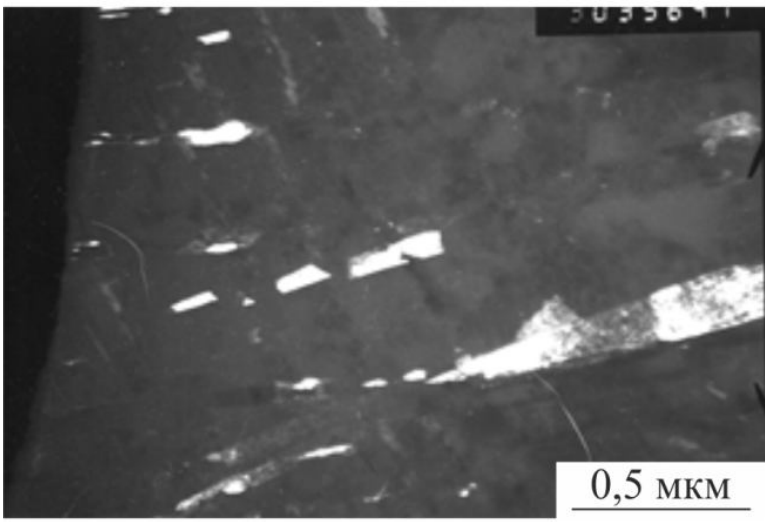

B

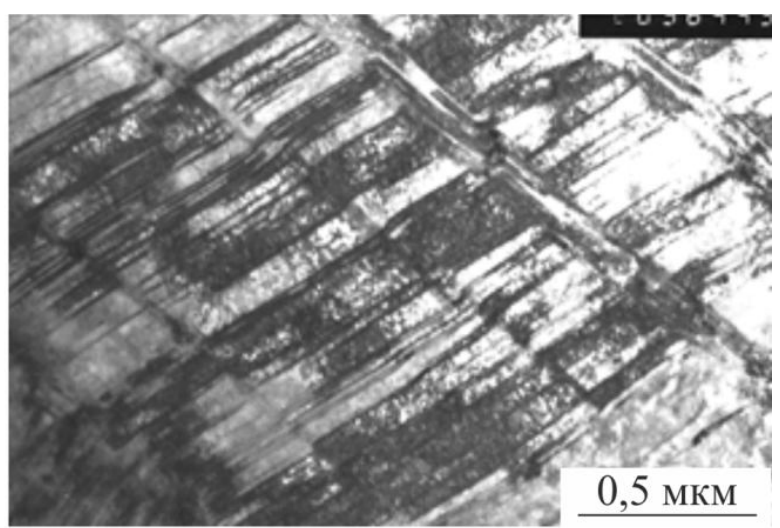

6

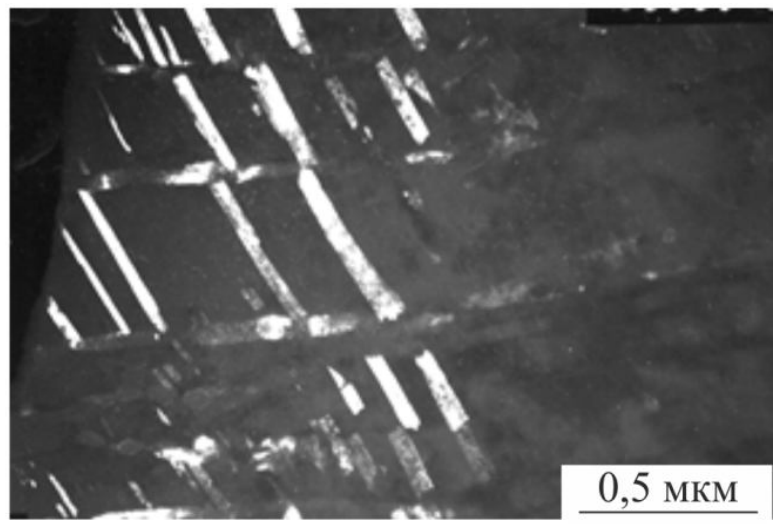

2

Рис. 4. Скопления микродвойников, принадлежащих к двум системам двойникования, в стали 12Х18Н10Т после нагружения: $a, \sigma$ - светлопольные изображения; 6 - темнопольное изображение в рефлексе $(111)_{\gamma} ;$ г - темнопольное изображение в рефлексе $(002)_{\gamma}$

В местах пресечения микродвойников принадлежащих к разным системам двойникования происходит сложное изменение ориентации кристаллической решетки $\gamma$-фазы. Ранее в работах [2-3] отмечалось, что в таких местах происходит образование $\alpha$-фазы. О возможности появления $\alpha$-фазы в местах пересечения полос сдвига сообщалось в работах $[10,11]$.

В работе [12] при квазисферическом нагружении шаровых образцов из стали $12 \mathrm{X} 18 \mathrm{H} 10 \mathrm{~T}$, имеющей в исходном состоянии карбидную полосчатость, было обнаружено, что дисперсные кристаллы мартенсита образуются в полосах с карбидами. Из этого факта авторы сделали заключение, что частицы карбидов инициируют образование разнонаправленных двойников при ударно-волновом нагружении и при взаимодействии двойников в полосах с карбидами образуется мартенсит. Однако в нашем случае карбидная полосчатость в исходном состоянии отсутствовала. Поэтому нельзя связывать образование $\alpha$-фазы с карбидной полосчатостью.

В работе [5] отмечалось, что при ударном нагружении превращение $\gamma \rightarrow \alpha$ протекает очень слабо и количество выделений $\alpha$-фазы сильно зависит от длительности импульса давления. Более продолжительный импульс создает более благоприятные условия для роста частиц $\alpha$-фазы. Таким образом, небольшое количество образующейся $\alpha$-фазы в исследованных 
образцах можно объяснить малой продолжительностью импульса нагрузки в проведенных экспериментах.

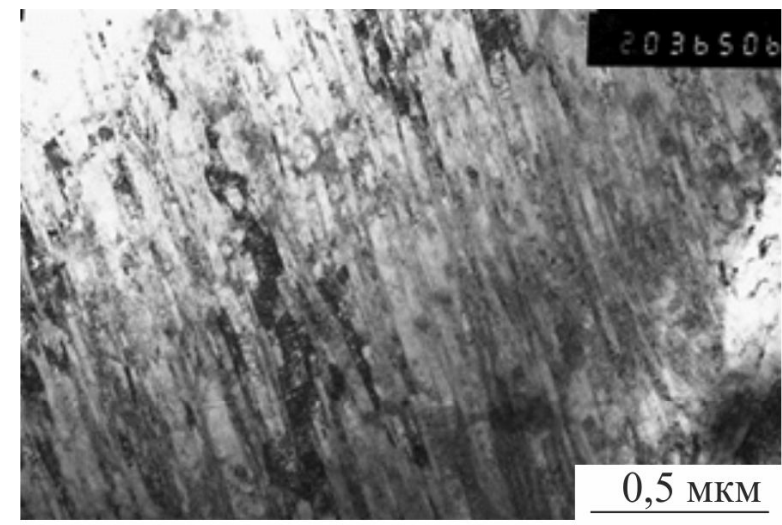

$a$

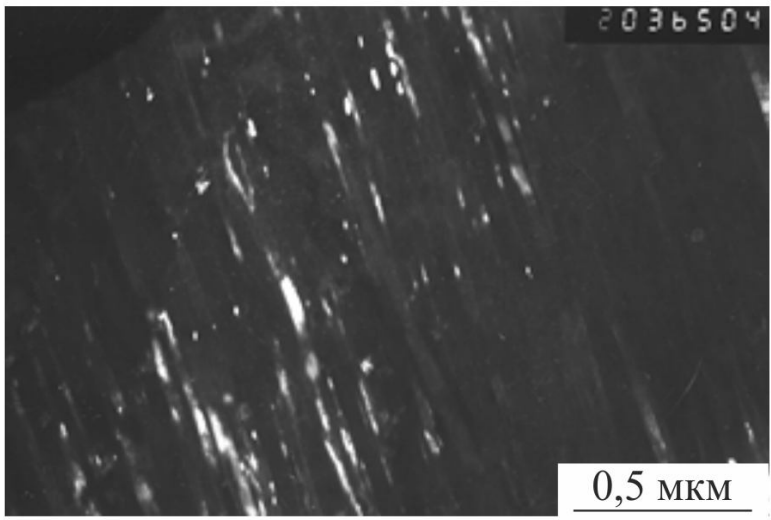

B

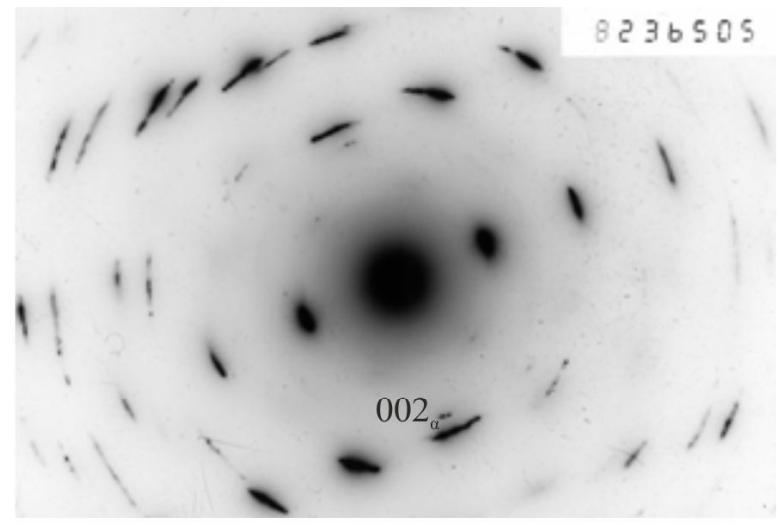

$\sigma$

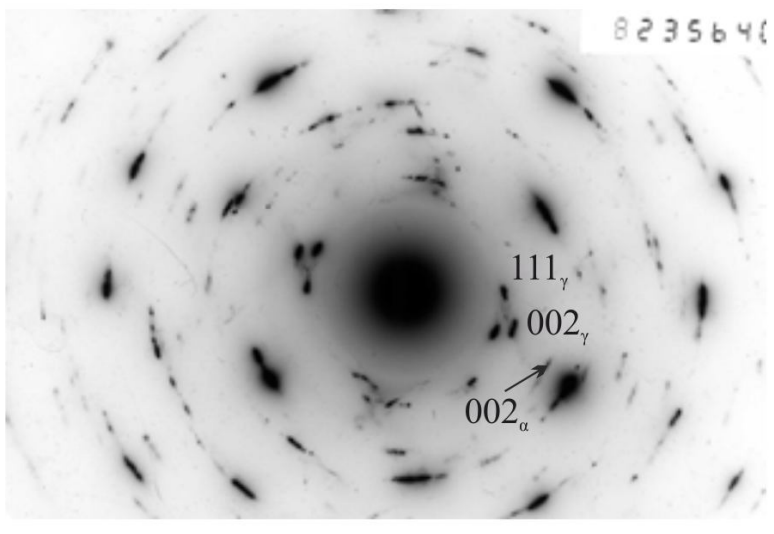

2

Рис. 5. Выделения $\alpha$-фазы, образовавшиеся в стали $12 \mathrm{X} 18 \mathrm{H} 10 \mathrm{~T}$ после ударного нагружения: $a$ - светлопольное изображение; $\sigma$ - микроэлектронограмма к $(a)$, ось зоны [112]; в - темнопольное изображение, полученное в рефлексе (002) 2- микроэлектронограмма к рис. 4 в, 2

\section{4. Заключение}

Проведенное исследование показало, что под действием сферически сходящихся ударных волн происходит сильная локализация пластической деформации, приводящая к образованию большого количества грубых следов скольжения. Образование нескольких неэквивалентных систем скольжения в отдельных зернах указывает на то, что в процессе взрывного нагружения критическое напряжение сдвига одновременно достигается в нескольких системах. Наряду со скольжением основным механизмом высокоскоростной пластической деформации стали 12X18Н10Т является также двойникование. В условиях взрывного нагружения двойникование протекает очень интенсивно, приводя к образованию протяженных областей, состоящих из большого количества близко расположенных микродвойников.

Фазовое $\gamma \rightarrow \alpha$ превращение в процессе взрывного нагружения протекает в обеих оболочках независимо от их толщины. Образующаяся $\alpha$-фаза в структуре нержавеющей стали наблюдается в виде мелких выделений.

\section{Благодарность}

Работа выполнена в рамках государственного задания ФАНО России (тема «Деформация» № 01201463327) при частичной поддержке проекта УрО РАН № 15-17-2-11. 
Электронно-микроскопическое исследование выполнено в ЦКП «Испытательный центр нанотехнологий и перспективных материалов» ИФМ УрО РАН.

Выражаем благодарность Е. А. Козлову начальнику отдела РФЯЦ-ВНИИТФ за предоставление оболочек для исследования.

\section{Литература}

1. Meyers M. F., Murr L. E. Defect generation in shock-wave deformation // Shock waves and high-strain-rate phenomena in metals / M. A. Meyers, L. E. Murr, eds. - New York: Plenum Press. - 1981. P. 487-530.

2. Sencer B. H., Maloy S. A., Gray III G. T. The influence of explosive-driven shock prestraining at $35 \mathrm{GPa}$ and of high deformation on the structure/property behavior of $316 \mathrm{~L}$ austenitic stainless steel// Metallurgical and Materials Transactions A: Physical Metallurgy and Materials Science. - 2005. - Vol. 36, iss. 7. - P. 1825-1831. - DOI: 10.1016/j.actamat.2005.03.037.

3. Lee Woel-Shyan, Lin Chi-Feng. Comparative study of the impact response and microstructure of 304L stainless steel with and without prestrain // Metallurgical and Materials Transactions A. - 2002. - Vol. 33, iss. 9. - P. 2801-2810. - DOI: 10.1007/s11661-002-0265-4.

4. Murr L. E., Staudhammer K. P., Hecker S. S. Effects of Strain State and Strain Rate on Deformation-Induced Transformation in 304 Stainless Steel: Part II. Microstructural Study // Metallurgical Transactions A. - 1982. - Vol. 13, iss. 4. - P. 627-635. - DOI: 10.1007/BF02644428.

5. The influence of explosive-driven "taylor-wave" shock prestraining on the structure/property behavior of 304 stainless steel / S. A. Malloy, G. T. Gray III, C. M. Cady, R. W. Rutherford, R. S. Hihson // Metallurgical and Materials Transactions A. - 2004. - Vol. 35, iss. 9. - P. 2617-2624. - DOI: 10.1007/s11661-004-0207-4.

6. Mechanical twins in 304 stainless steel after small-charge explosion / D. Firraro, P. Matteis, G. Scavino, G. Ubertalli, M. G. Ienco, G. Pellati, P. Piccardo, M. R. Pinasco, E. Stagno, R. Montanari, M. E. Tata, G. Brandimarte, S. Petralia // Materials Science and Engineering: A. 2006. - Vol. 424, iss 1-2, P. 23-32. DOI: 10.1016/j.msea.2006.02.036.

7. Special features in convergence dynamics of steel shells under their explosive loading. Results of laser-interferometric measurements / E. A. Kozlov, S. A. Brichikov, D. S. Boyarnikov, D. P. Kuchko, A. A. Degtyarev // The Physics of Metals and Metallography. - 2011. - Vol. 112, iss. 4. - P. 389-404. - DOI: 10.1134/S0031918X11040259.

8. Rutkowska-Gorczyca M., Podrez-Radziszwska M., Kajtoch J. Corrosion resistance and microstructure of steel AISI 316L after cold plastic deformation // Metallurgy and foundry engineering. - 2009. - Vol. 35, no. 1. - P. 35-42.

9. Borodin E. N., Atroshenko S. A., Mayer A. E. Distribution of dislocations and twins in copper and 18Cr-10Ni-Ti steel under shock-wave loading // Technical Physics. - 2014. - Vol. 59, iss. 8. - P. 1163-1170. - DOI: 10.1134/S1063784214080076.

10. Bogers A. J., Burgers W. G. Partial dislocations on the $\{110\}$ planes in the B.C.C. lattice and the transition of the F.C.C. into the bec lattice // Acta Metallurgica. - 1964. - Vol. 12, iss. 2. P. 255-261. - DOI: 10.1016/0001-6160(64)90194-4.

11. Talonen J., Hanninen H. Formation of shear bands and strain-induced martensite during plastic deformation of metastable austenitic stainless steels // Acta Materialia. - 2007. - Vol. 55, iss. 18. - P. 6108-6118. - DOI: 10.1016/j.actamat.2007.07.015.

12. Formation of martensite in austenitic steel upon loading by quasi-spherical converging shock waves / V. I. Zel'dovich, A. E. Kheifets, N. Yu. Frolova, A. K. Muzyrya, A. Yu. Simonov // The Physics of Metals and Metallography. - 2013. - Vol. 114, iss. 12. - P. 1031-1037. DOI: $10.1134 / \mathrm{S} 0031918 \mathrm{X} 13120090$. 